\title{
High- and low-frequency phonon modes in dipolar quantum gases trapped in deep lattices
}

\author{
Aleksandra Maluckov ${ }^{1}$, Goran Gligorić ${ }^{1}$, Ljupčo Hadžievski ${ }^{1}$, Boris A. Malomed ${ }^{2}$, and Tilman Pfau ${ }^{3}$ \\ 1 Vinča Institute of Nuclear Sciences, University of Belgrade, P. O. B. 522,11001 Belgrade, Serbia \\ 2 Department of Physical Electronics, School of Electrical Engineering, \\ Faculty of Engineering, Tel Aviv University, Tel Aviv 69978, Israel \\ ${ }^{3}$ Physikalisches Institut, Universität Stuttgart, Pfaffenwaldring 57, 70569 Stuttgart, Germany
}

\begin{abstract}
We study normal modes propagating on top of the stable uniform background in arrays of dipolar Bose-Einstein condensate (BEC) droplets trapped in a deep optical lattice. Both the on-site meanfield dynamics of the droplets and their displacement due to the repulsive dipole-dipole interactions (DDIs) are taken into account. Dispersion relations for two modes, viz., high- and low- frequency counterparts of optical and acoustic phonon modes in condensed matter, are derived analytically and verified by direct simulations, for both cases of the repulsive and attractive contact interactions. The (counterpart of the) optical-phonon branch does not exist without the DDIs. These results are relevant in the connection to emerging experimental techniques enabling real-time imaging of the condensate dynamics and direct experimental measurement of phonon dispersion relations in BECs.
\end{abstract}

PACS numbers: 03.75.Lm; 05.45.Yv; 63.20.-e; 63.20.Dj

\section{INTRODUCTION}

The propagation of collective excitations in periodically structured media has been recognized long ago as a fundamentally important topic of condensed-matter physics [1]. More recently, it has been understood that many condensed media, where the intrinsic dynamics is very complex, may be effectively "simulated" by rarefied quantum gases, for which it is much easier to predict and observe fundamental dynamical effects [2, 3]. In the latter context, the role of the periodic structure is commonly played by optical-lattice (OL) potentials [4]. In particular, the use of atomic Bose-Einstein condensates (BECs) trapped in OLs makes it possible to simulate the propagation of acoustic waves in crystals theoretically [5]- 7 ] and in a direct experiment [8].

A deep OL potential splits the condensate into an array of droplets trapped in local potential wells, which are weakly coupled with nearest neighbors by tunneling of atoms across potential barriers. In this case, it is natural to approximate the global BEC wave function by a superposition of wavelets of locally trapped atoms, which resemble well-known Wannier modes that can be used an alternative basis instead of Bloch waves [9]. On the basis of this expansion, the reduction of the underlying full Gross-Pitaevskii equation (GPE) to its discrete version (DGPE) has been rigorously derived in several works [10] and reviewed in Ref. [11]. The derivation of the DGPE was further extended to the model with a relatively strong contact nonlinearity, when the onsite nonlinearity in the resulting discrete equation is nonpolynomial 12 .

A significant extension of the variety of dynamical effects in atomic BECs is provided by long-range dipole-dipole interactions (DDIs). Basic and more sophisticated properties of dipole condensates were predicted theoretically [16], which was followed by the creation of such condensates in vapors of ${ }^{52} \mathrm{Cr}$ [17]. The flexibility of this setting is buttressed by the possibility to vary the relative strength of the DDI and contact interactions by means of the Feshbach resonance affecting the scattering length for colliding chromium atoms [18]. Results obtained in this area have been reviewed in Ref. [19].

Further progress in the experimental work has recently resulted in the creation of dipolar condensates with the strongest possible atomic DDIs in ${ }^{164}$ Dy [20]. Also promising is the work with erbium [21], and with gases composed of molecules carrying electric dipole moments 22 ].

OLs provide a powerful set of tools for the studies of dipolar BECs. In particular, the stabilization and destabilization of the condensate, trapped in deep OLs, by DDI was demonstrated theoretically and experimentally [23]. It was straightforward to generalize the DGPE for this case, which leads to the discrete equation combining the local onsite nonlinearity with the nonlocal nonlinear interaction between the sites, in the one- 13, 14] and two-dimensional (2D) [15] settings alike. Further, we have recently demonstrated that, in case the uniform (alias continuous-wave, $\mathrm{CW}$ ) state of the dipolar condensate, trapped in a deep periodic potential (i.e., the discretized state with equal amplitudes at all sites of the lattice), is modulationally unstable, two- and three-period density patterns emerge as energy minimizers, the stability area being very large for the three-period pattern [24]. Modulated-density solutions are also produced by the DGPE for condensates fragmented by the deep OL potential in the absence of long-range interactions, but in that case they are completely unstable [25]. 2D supersolid structures supported by the OL in the dipolar BEC were predicted too [26], and anisotropic DDIs may give rise to striped 2D patterns even in the absence of the lattice [27]. 
Beyond the aforementioned detailed studies of static patterns, a physical problem of obvious interest is the study of collective excitations propagating on top of stable states supported by the long-range interactions. In this context, a well-known result is the prediction of the roton branch of excitations in the dipolar condensates 28]. The subject of the present work is the analysis of excitation modes propagating through the dipolar BEC fragmented by the deep OL and, accordingly, modeled by the DGPE including the long-range DDIs. The rapid progress in experimental studies of condensates (in particular, the development of techniques for in-situ imaging of the condensate dynamics [29, 30]) suggests that the direct observation of such modes will become possible, hence the analysis is a subject of direct relevance. A related promising experimental technique, based on the method of the Bragg spectroscopy [31], was very recently elaborated for directly measuring phonon dispersion relations in trapped BECs [30]. In this work, we focus on the most fundamental case of the normal modes excited on top of the stable CW state. Still more challenging situations with the underlying periodically modulated density profiles will be considered elsewhere.

The most essential result of the analysis is that the long-range DDI between condensate droplets gives rise to a highfrequency branch of collective excitations, which emulates the optical-phonon mode well known in condensed-matter physics. This excitation propagates as a displacement wave in the array of trapped BEC droplets, which resembles the dynamics of the Frenkel-Kontorova model [32]. Another fundamental branch of the propagating waves represents low-frequency density oscillations of the droplets, which is a counterpart of acoustic phonons in condensed matter. It is a generalization of the branch which was recently found in the limit of immobile droplets 24].

Excitation modes similar to those reported here may be expected in other physical settings which feature long-range interactions between elements of a fragmented wave field. In particular, laser illumination of atoms may induce an effective gravity-like long-range attraction in BEC, which, in the combination with the OL, may give rise to a supersolid [33]. An example in optics is offered by the light field trapped in an arrayed waveguide embedded into a medium with the nonlocal thermal nonlinearity [34], which gives rise to a number of patterns in one and two dimensions, as demonstrated theoretically [35] and experimentally [36]. Other possible realizations of this generic setting in optics may use the nonlocal nonlinearity of liquid crystals [37], and an effective nonlocality in semiconductor waveguides induced by the transport of charge carriers [38].

\section{THE MODEL}

The on-site dynamics of the dipolar-BEC droplets trapped in local potential wells of the deep OL potential is governed by the DGPE, which includes the onsite cubic nonlinearity and long-range DDI. Following the notation adopted in Ref. [24], the discrete equation is written as

$$
i \frac{d f_{n}}{d t}=-C_{n}\left(f_{n+1}-f_{n}\right)-C_{n-1}\left(f_{n-1}-f_{n}\right)+\sigma\left|f_{n}\right|^{2} f_{n}-\Gamma \sum_{m \neq n} \frac{\left|f_{m}\right|^{2}}{\left|x_{m}-x_{n}\right|^{3}} f_{n},
$$

where $f_{n}$ is the discrete wave function, $C_{n}$ are inter-site coupling coefficients proportional to the overlapping integrals between the Wannier modes exponentially localized at adjacent sites [10]- 13]. If the coordinate of the $n$-th droplet is taken as $x_{n}=n+\xi_{n}$, where $\xi_{n}$ accounts for its displacements due to the DDIs with other droplets (see Fig. 11), then the corresponding dependence of coefficients $C_{n}$ in Eq. (10) on $\xi_{n}$ is

$$
C_{n}=C^{(0)} \exp \left(-\frac{1+\xi_{n+1}-\xi_{n}}{l}\right) \equiv c \exp \left(-\frac{\xi_{n+1}-\xi_{n}}{l}\right)
$$

$c \equiv C^{(0)} \exp (-1 / l)$, and $C^{(0)}$ is a coefficient of tunneling of atoms between adjacent sites separated by distance $l$.

Proceeding to nonlinear terms in Eq. (11), $\sigma=-1$ and +1 correspond to the attractive or repulsive onsite contact interaction (abbreviated below as AC or RC, respectively), and $\Gamma$ is the relative strength of the DDI, with respect to the onsite nonlinearity [13]. In the present notation, $\Gamma>0$ and $\Gamma<0$ correspond to the attractive and repulsive DDIs, respectively. According to its definition, $\Gamma$ may be adjusted by means of the Feshbach resonance which affects the strength of the local nonlinearity, and, additionally, by fixing the orientation of the magnetic moments with respect to the system's axis.

The energies of the DDI, and of the trapping of the condensate in the OL potential are

$$
U_{\mathrm{DD}}=-\Gamma \sum_{m \neq n} \frac{\left|f_{m}\right|^{2}\left|f_{n}\right|^{2}}{\left|x_{n}-x_{m}\right|^{3}}, U_{\mathrm{OL}}=-U_{0} \sum_{n} \cos \left(2 \pi x_{n}\right),
$$

where $U_{0}$ is the depth of the periodic potential, hence the motion of each droplet is governed by the Newton's equation,

$$
\rho \frac{d}{d t}\left(\left|f_{n}\right|^{2} \frac{d \xi_{n}}{d t}\right)=-\frac{\partial}{\partial \xi_{n}}\left(U_{\mathrm{DD}}+U_{\mathrm{OL}}\right),
$$


where $\rho$ is the density of the condensate, so that the mass and momentum of the $n$-th droplet are $\rho\left|f_{n}\right|^{2}$ and $\rho\left|f_{n}\right|^{2} d \xi_{n} / d t$, as shown in Fig. 1].

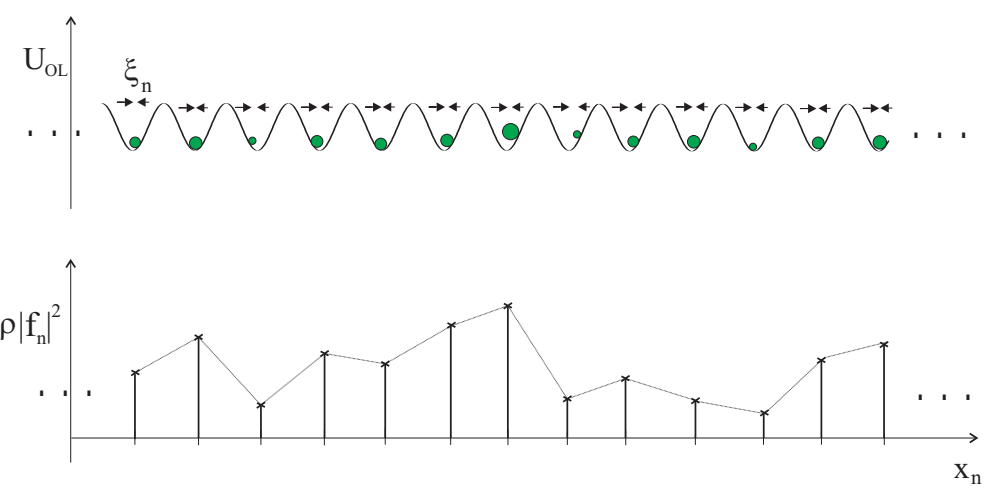

FIG. 1: (Color online) In the top panel, circles represent the array of droplets trapped in the OL potential. The bottom panel displays a generic distribution of droplet masses, which are proportional to areas of the circles.

Thus, the dynamical system following from Eqs. (11), (4) and (3) takes the following form:

$$
\begin{gathered}
i \frac{d f_{n}}{d t}=-c\left[e^{-\frac{\xi_{n+1}-\xi_{n}}{l}}\left(f_{n+1}-f_{n}\right)+e^{-\frac{\xi_{n}-\xi_{n-1}}{l}}\left(f_{n-1}-f_{n}\right)\right] \\
+\sigma\left|f_{n}\right|^{2} f_{n}-\Gamma \sum_{m \neq n} \frac{\left|f_{m}\right|^{2}}{\left|n-m+\xi_{n}-\xi_{m}\right|^{3}} f_{n} \\
\rho \frac{d}{d t}\left(\left|f_{n}\right|^{2} \frac{d \xi_{n}}{d t}\right)=-2 \pi U_{0} \sin \left(2 \pi \xi_{n}\right) \\
+3 \Gamma\left|f_{n}\right|^{2}\left(\sum_{m>n}-\sum_{m<n}\right)\left(\frac{\left|f_{m}\right|^{2}}{\left|n-m+\xi_{n}-\xi_{m}\right|^{4}}\right) .
\end{gathered}
$$

This system was simulated by means of the Runge-Kutta method of the sixth order.

\section{NORMAL EXCITATION MODES}

To study collective-excitation modes in the present context, we set $f_{n}=\left(\phi_{0}+g_{n}\right) e^{-i \mu t}$, where $\phi_{0}=\sqrt{\mu /[\sigma-2 \Gamma \zeta(3)]}$ is the amplitude of the CW state, as found in Ref. [24] [ $\zeta(3) \approx 1.2021$ is the value of the Riemann's zeta-function], and linearize Eqs. (5) and (6) with respect to small perturbations, $g_{n}$ and $\xi_{n}$ :

$$
\begin{gathered}
i \frac{d g_{n}}{d t}=\left[-\mu+2 c+2(\sigma-2 \Gamma \zeta(3)) \phi_{0}^{2}\right] g_{n}-c\left(g_{n+1}+g_{n-1}\right) \\
-\Gamma \phi_{0}^{2} \sum_{q>0} q^{-3} \Pi_{q}+\sigma \phi_{0}^{2} g_{n}^{*}+3 \phi_{0}^{3} \Gamma \sum_{q>0} q^{-4}\left(\xi_{n+q}-\xi_{n-q}\right) \\
\rho \frac{d^{2} \xi_{n}}{d t^{2}}=\left[-\left(\frac{2 \pi}{\phi_{0}}\right)^{2} U_{0}-24 \Gamma \phi_{0}^{2} \sum_{q>0} q^{-5}\right] \xi_{n} \\
+24 \Gamma \phi_{0}^{2} \sum_{q>0} q^{-5}\left(g_{n-q}^{*}-g_{n+q}^{*}+g_{n-q}^{*}-g_{n+q}^{*}\right)
\end{gathered}
$$

where $q$ is an integer index of the summation over all sites of the lattice, the asterisk stands for the complex conjugate, and $\Pi_{q} \equiv g_{n-q}+g_{n+q}+g_{n-q}^{*}+g_{n+q}^{*}$. Difference-differential equations (7) are Fourier-transformed into the system of algebraic equations,

$$
\left[\begin{array}{ccc}
A+\omega & -B & -i F \\
-B & A-\omega & -i F \\
i\left(\rho \phi_{0}\right)^{-1} F & i\left(\rho \phi_{0}\right)^{-1} F & D-\omega^{2}
\end{array}\right]\left[\begin{array}{c}
G_{k}(\omega) \\
G_{-k}^{*}(-\omega) \\
\Xi_{k}(\omega)
\end{array}\right]=0
$$


where $G_{k}(\omega)$ and $\Xi_{k}(\omega)$ are the Fourier transforms of $g_{n}(t)$ and $\xi_{n}(t)$, and

$$
\begin{aligned}
A & \equiv-4 c \sin ^{2}\left(\frac{k}{2}\right)-B, B \equiv \phi_{0}^{2}\left[\sigma-2 \Gamma \sum_{q>0} \frac{\cos (k q)}{q^{3}}\right], \\
F & \equiv 6 \Gamma \phi_{0}^{2} \sum_{q>0} \frac{\sin (k q)}{q^{4}}, \\
D & \equiv \frac{1}{\rho}\left\{\left(\frac{2 \pi}{\phi_{0}}\right)^{2} U_{0}+24 \Gamma \phi_{0}^{2}\left[\zeta(5)-\sum_{q>0} q^{-5} \cos (k q)\right]\right\},
\end{aligned}
$$

with $\zeta(5) \approx 1.0369$.

Dispersion relations for propagating waves, $\omega=\omega(k)$, are derived from Eq. (8) by equating the determinant of the system's matrix to zero, which yields a biquadratic equation,

$$
\omega^{4}-\left(A^{2}-B^{2}+D\right) \omega^{2}+D\left(A^{2}-B^{2}\right)-\frac{2 F^{2}}{\rho \phi_{0}}(A+B)=0 .
$$

In the parameter region of interest, which corresponds to the stable CW background, Eq. (10) yields four solutions for the frequency, $\omega(k)=\left\{\omega_{1}(k), \omega_{2}(k)=-\omega_{1}(k), \omega_{3}(k), \omega_{4}(k)=-\omega_{3}(k)\right\}$. In the following we display the positivefrequency branches, the negative ones being their specular images. Further straightforward analysis of the solutions produced by Eq. (8) demonstrates that the low- and high-frequency branches represent waves of onsite density oscillations and droplet displacements, respectively. The latter one may also be called a dipolar-phonon mode.

The BEC system which does not admit the droplet displacements (in other words, the corresponding trapping frequency is assumed to be infinite) is modeled by the first equation in (6) with $\xi_{n} \equiv 0$ [24]. The corresponding dispersion relation amounts to the single positive real root for $\omega^{2}$ in the parameter region where the CW background is stable. It represents the density-oscillation mode in the case of frozen displacements.

The modes in the present system may be compared to Langmuir and ion-acoustic waves in plasmas [39]. In this context, the displacement mode, although it originates as the dipolar-phonon wave, is a counterpart of the highfrequency Langmuir wave of electron oscillations in the plasma, while the density-oscillation mode is an analog of the ion sound. The reason for this "switch of the roles" is that the displacement wave is actually a stream of optical phonons, in terms of solid state [1], while the ion sound in the plasma is carried by acoustic phonons. Further, in the long-wave limit the dispersion relation for the low-frequency density mode takes precisely the form of the $\omega(k)$ relation for acoustic phonons, see Eq. (13) below.

\section{RESULTS AND DISCUSSION}

\section{A. Analytical considerations}

The analysis of stability conditions for the CW background in the present system demonstrates that they are identical to those in the previously considered model [24] with the frozen displacement degree of freedom. For the RC interactions $[\sigma=+1$ in Eq. (10)], the CW-stability region extends to arbitrarily weak repulsive DDIs, $\Gamma \leq 0$ (see Fig. 1 in Ref. [24]), while in the case of the AC interactions $(\sigma=-1)$ the $\mathrm{CW}$ has a stability region if the repulsive DDI is strong enough,

$$
|\Gamma|>\left|\Gamma_{\text {thr }}\right|,
$$

with the threshold value that can be found from dispersion relation (10). For $\mu=1$ (the chemical potential which is adopted in figures displayed here), $\Gamma_{\text {thr }} \approx-1.27$. To relate these results to the experimental situation, it is relevant to mention that as shown in Ref. [13], a typical value $|\Gamma|=0.5$, relevant to our analysis (see Fig. 2 below), corresponds to parameter values which are realistic in experiments with ${ }^{52} \mathrm{Cr}$ : the scattering length $\simeq 2 \mathrm{~nm}$ and the transverseconfinement radius $\simeq 5 \mathrm{~mm}$. Actually, positive and negative values of $\Gamma$ may be varied within broad limits by means of the Feshbach resonance, as shown experimentally [17].

As mentioned above, the analytical solution of dispersion equation (10) demonstrates that the stable CW background supports two modes of the propagating excitations, viz., the low-frequency acoustic-phonon and high-frequency opticalphonon modes. The two dispersion curves are displayed in Fig. 2, for both signs of the contact interaction, RC and AC. A noteworthy peculiarity of the latter (AC) case is that the dispersion relation has a minimum at the center of 

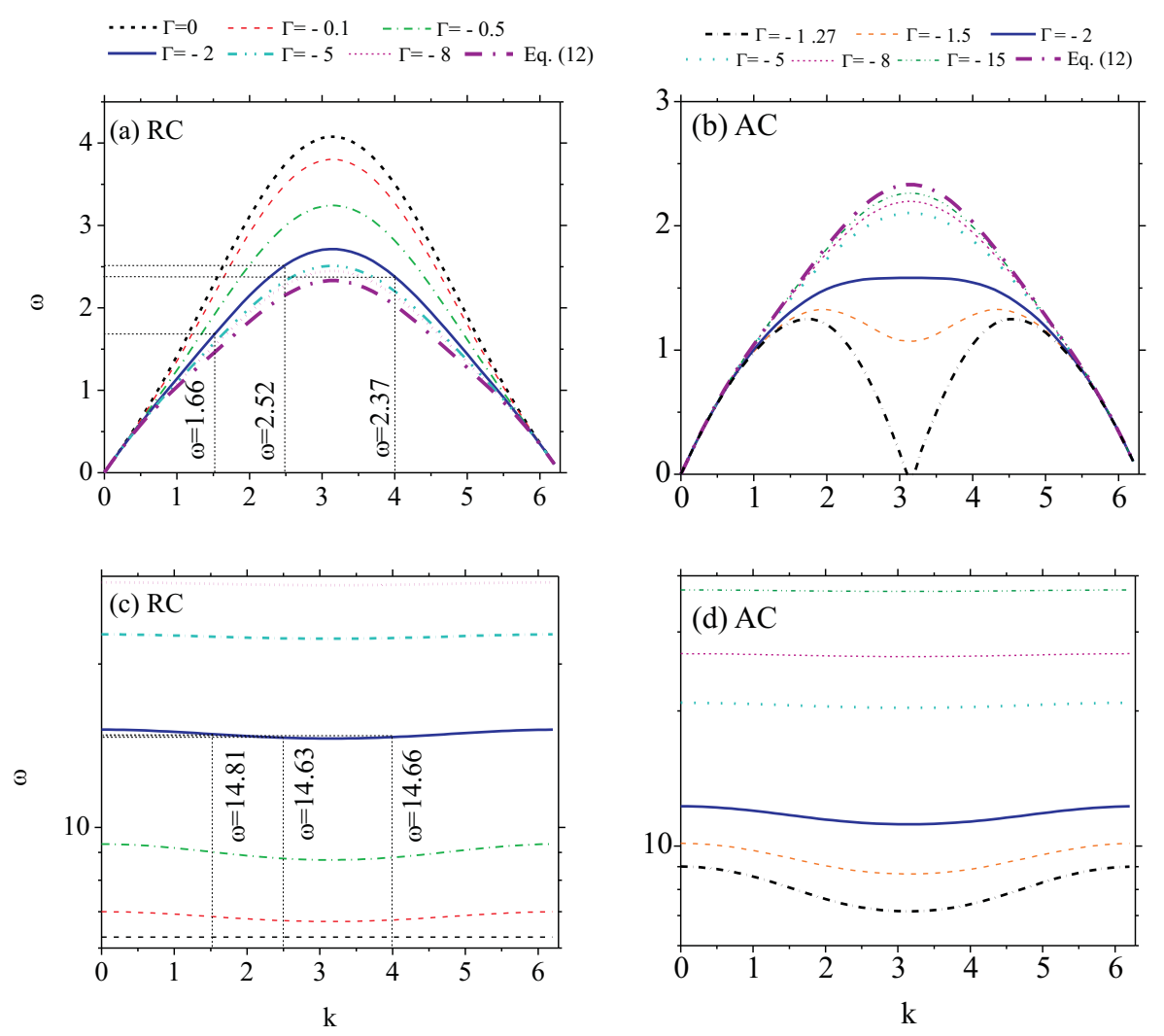

FIG. 2: (Color online) The top row: dispersion curves for the low-frequency acoustic phonon mode, with repulsive (a) and attractive (b) signs of the contact interaction ( $\mathrm{RC}$ and $\mathrm{AC}$, respectively). The bottom row: dispersion curves for the highfrequency optical-phonon mode, with the RC (c) and AC (d) signs of the local nonlinearity. The curves are drawn for $\mu=1$, in the first Brillouin zone, $0 \leq k<2 \pi$. The corresponding relative values of the DDI strength, $\Gamma$, are indicated in the plots. Vertical lines in panels (a) and (c) indicate frequencies predicted by the two dispersion curves at three wavenumbers, $k=1.57$, 2.5, and 4, for which numerically computed temporal spectra of lattice perturbations, excited as per Eq. (15), are displayed below in Fig. 4

the Brillouin zone, provided that the repulsive DDI is relatively weak. We stress that only the situations with the stable CW background are shown, therefore values of $\Gamma$ which do not satisfy stability condition (11) are not included in panel 2(b).

The decrease of the largest frequency of the acoustic-phonon mode with the increase of $|\Gamma|$, which is observed in Fig. 2(a), implies that the system becomes "less stiff", for the propagation of the density waves, with the strengthening of the repulsive DDI. A reason for this counter-intuitive conclusion is that the model does not directly take into account the DDI inside each droplet. However, this may be included via a renormalization of the onsite contact interaction. On the other hand, Fig. 2(b) demonstrates that the stiffness increases with $|\Gamma|$ in the case of the AC interactions $(\sigma=-1)$. In either cases of the $\mathrm{RC}$ or $\mathrm{AC}$ interactions, with the increase of $|\Gamma|$ the dispersion curves for the low-frequency (LF) acoustic-phonon mode asymptotically approach a limit form which can be derived from Eqs. (9) and (10):

$$
\omega_{\mathrm{LF}}(\Gamma \rightarrow-\infty)= \pm 4 c \sin \left(\frac{k}{2}\right) \sqrt{\sin ^{2}\left(\frac{k}{2}\right)+\frac{\mu}{2 c \zeta(3)} \sum_{q>0} q^{-3} \cos (k q)} .
$$

In Figs. 2(a) and (b), the dispersion curves approach the asymptotic one from above and below in the cases of the $\mathrm{RC}$ and $\mathrm{AC}$ interactions, respectively.

Naturally, these results imply that the acoustic-phonon mode can propagate in the absence of the DDI $(\Gamma=0)$ in the condensate with the RC interactions only. The addition of the DDIs modifies the corresponding dispersion curve 

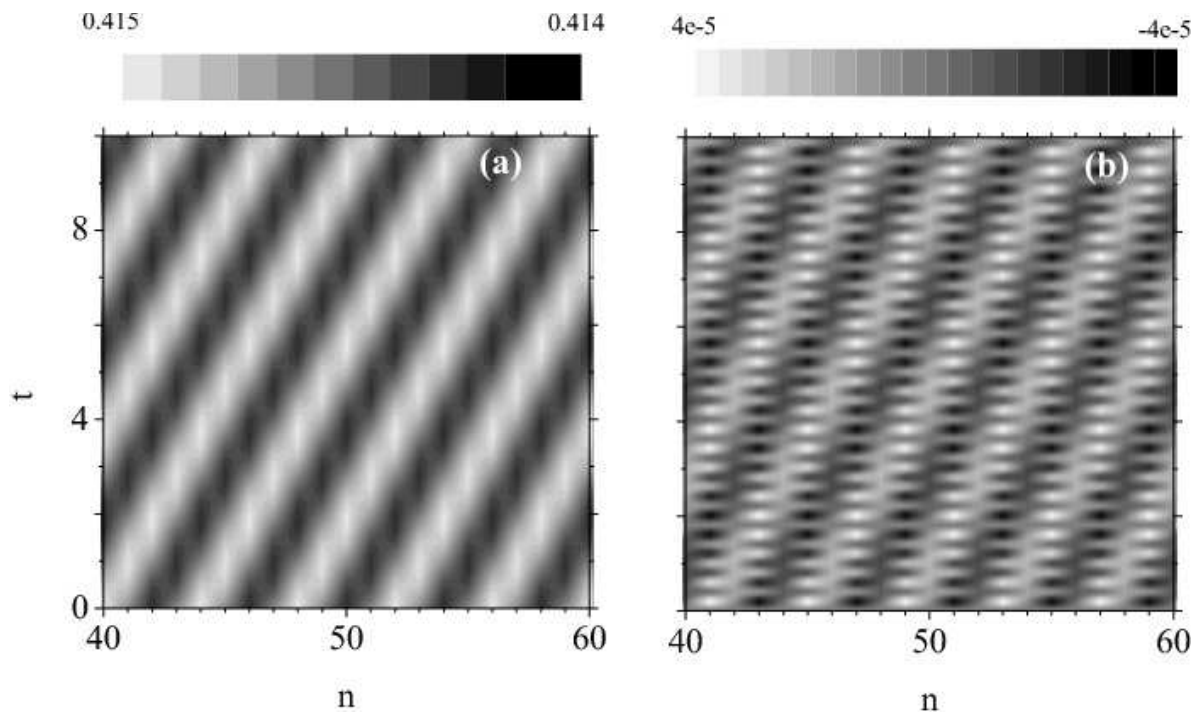

FIG. 3: (Color online) Contour plots of the density, $\left|f_{n}\right|$ (a), and displacement, $\xi_{n}$ (b), for simultaneously excited acoustic and optical phonon waves, in the case of the repulsive contact and dipole-dipole interactions, $\sigma=+1$ and $\Gamma=-2$. The wavenumber and amplitude of the initial excitation (15) are $k=\pi / 2=1.57$ and $\varepsilon=0.001$. The respective frequencies of the propagating low- and high-frequency modes are $\omega_{\mathrm{LF}}=1.8(\mathrm{a})$, and $\omega_{\mathrm{HF}}=14.9(\mathrm{~b})$, which agree with those obtained from dispersion relations (10) and the temporal spectra (see Fig. 4).

without altering it qualitatively. Note also that the acoustic-phonon mode curve takes the form of $\omega \approx v k$ for small $k$, with the effective sound velocity that can be found from Eqs. (10) and (9):

$$
v=\phi_{0} \sqrt{2 c(\sigma-2 \Gamma \zeta(3))} .
$$

On the other hand, the optical-phonon mode cannot propagate in the absence of the repulsive DDI, i.e., at $\Gamma=0$, when it degenerates into uncoupled oscillations of the droplets around local minima of the lattice potential, with frequency $\omega_{\text {osc }}=\left(2 \pi / \phi_{0}\right) \sqrt{U_{0} / \rho}$ [see the horizontal black dotted line in Fig. 2 (c)]. Further, the expansion of the $\omega(k)$ relation for the high-frequency (HF) optical-phonon mode in the long-wave limit (for small $k^{2}$ ) exhibits a quadratic dispersion, similar to that for the Langmuir waves in the plasma:

$$
\omega_{\mathrm{HF}}(k) \approx \frac{2 \pi}{\phi_{0}} \sqrt{\frac{U_{0}}{\rho}}-\frac{3 \phi_{0}^{3} \zeta(3)}{\pi} \frac{|\Gamma|}{\sqrt{U_{0} \rho}} k^{2} .
$$

\section{B. Direct simulations}

The propagation of the waves through the stable CW background was tested by direct simulations of Eqs. (7). The system was initially excited by a perturbation in the form of

$$
f_{n}(t=0)=\phi_{0}+\varepsilon \exp (i k n)
$$

with small amplitude $\varepsilon$ and arbitrary wavenumber $k$. The simulations demonstrate a superposition of the acousticphonon and optical-phonon propagating modes, as shown in Fig. 3 .

Characteristic frequencies of both types of the propagating modes, as obtained from the direct simulations, almost precisely fit those predicted by the dispersion relations. This is shown in Fig. 4 by means of sample temporal spectra representing the propagating perturbations excited as per Eq. (15) with $\varepsilon=0.001$ and three different wavenumbers, $k=1.57,2.5$, and 4 , in the case of the RC interactions. For each $k$, the creation of the acoustic-phonon and optical-phonon modes is observed. The central frequencies, indicated in the plots, are very close to their counterparts predicted by the dispersion curves, which are marked by vertical lines in Figs. 2(a,c).

Additional simulations (not displayed here) demonstrate that the increase of the amplitude of the initial perturbation $(\varepsilon)$ naturally makes the spectra wider and more complex. 

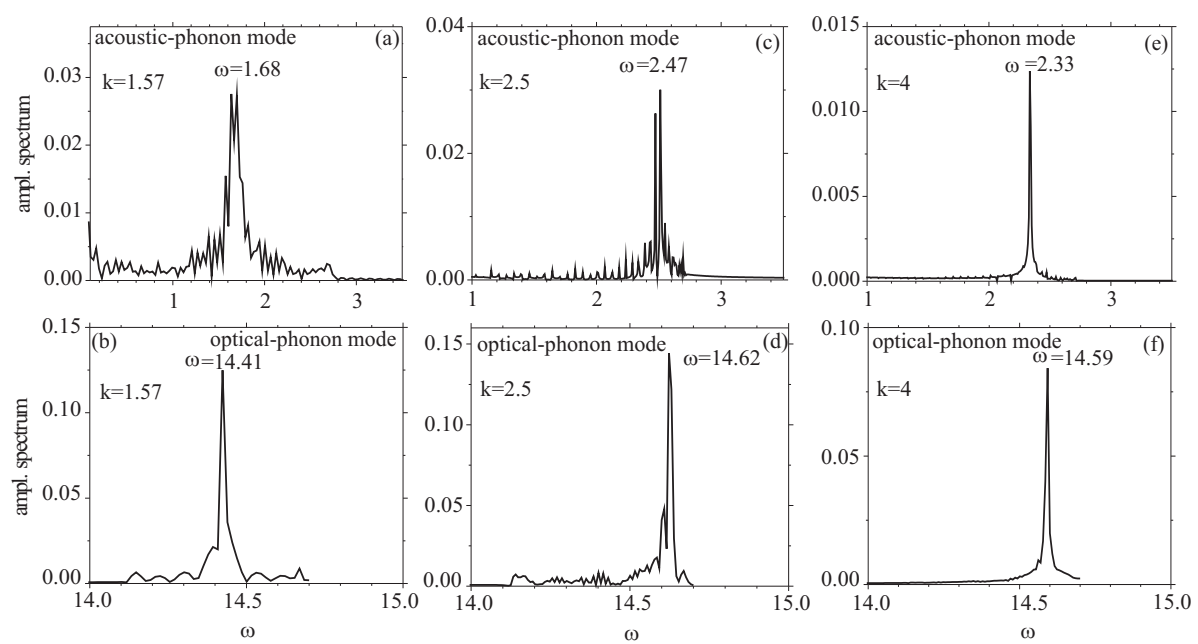

FIG. 4: Typical examples of temporal spectra of excitations in the system with $\sigma=+1, \Gamma=-2, \mu=1$, computed at a particular site of the lattice: (a), (c), (e) - the acoustic-phonon modes; (b), (d), (f) - the optical-phonon modes. The peak values of $\omega$ accurately fit their analytical counterparts obtained from Eq. (10), which are marked in Fig. 2

\section{CONCLUSIONS}

This work reports a new mode of high-frequency collective excitations propagating on top of the stable background in the dipolar BEC trapped in the form of the array of droplets in a deep OL potential. This branch of the excitations, which is similar to the optical-phonon mode in condensed matter, and also features similarity to Langmuir waves in plasmas, does not exist in the absence of the repulsive dipole-dipole interactions. The dispersion relations for the new mode and the additional low-frequency one of the acoustic-phonon type, which accounts for density oscillations in the trapped BEC, were derived analytically. The results have been confirmed by direct simulations of the excited system.

The currently developing techniques for in situ imaging of dynamical phenomena and recording phonon dispersion relations in trapped BEC [29, 30] open the way to experimental realization of the results reported in this work in atomic gases of chromium, dysprosium and erbium, and in molecular gases, such as KRb and NaLi. As concerns the theoretical analysis, it can be extended in several directions. First, it would be interesting to study the nonlinear dynamics of excitations with nonsmall amplitudes. Second, a natural extension is to consider collective excitations on top of stable periodically modulated patterns, such as the double- and triple-periodic ones 24]. This extension is expected to produce a larger number of different dynamical branches. Then, a challenging problem is to find normal modes of collective excitations in 2D settings. Finally, quite an interesting possibility is to introduce a deformable Bose-Hubbard model, as a quantum version of the mean-field system considered in the present work, which may be then implemented in chains of trapped ultracold atoms or ions 3].

A.M., G.G., and Lj.H. acknowledge support from the Ministry of Education and Science of Serbia (Project III45010). The work of B.A.M. and T.P. was supported in a part by the German-Israel Foundation through grant No. I-1024$2.7 / 2009$.

[1] R. E. Peierls, Quantum Theory of Solids (Oxford University Press: London, 1955).

[2] D. Jaksch and, P. Zoller, Ann. Phys. 315, 52 (2005); I. Bloch, J. Dalibard, and S. Nascimbène, Nature Phys. 8, 267 (2012); A. A. Houck, H. E. Türeci, and J. Koch, ibid. 8, 292 (2012); P. Hauke, F. M. Cucchietti, L. Tagliacozzo, I. Deutsch, and M. Lewenstein, Rep. Progr. Phys. 75, 082401 (2012).

[3] M. Johanning, A. F. Varón, and C. Wunderlich, J. Phys. B: At. Mol. Opt. Phys. 42, 154009 (2009).

[4] M. Greiner, O. Mandel, T. Esslinger, T. W. Hansch, and I. Bloch, Nature 415, 39 (2002); D. Jaksch, C. Bruder, J. L. Cirac, C. W. Gardiner, and P. Zoller, Phys. Rev. Lett. 81, 3108 (1998); V. A. Brazhnyi and V. V. Konotop, Mod. Phys. Lett. B 18, 627 (2004); O. Morsch and M. Oberthaler, Rev. Mod. Phys. 78, 179 (2006); M. Lewenstein, A. Sanpera, V. Ahufinger, B. Damski, A. Sen(De), and U. Sen, Adv. Phys. 56, 243 (2007); Bloch, J. Dalibard, and W. Zwerger, Rev. Mod. Phys. 80, 929 (2008).

[5] A. Smerzi and A. Trombettoni, Phys. Rev. A 68, 023613 (2003).

[6] C. Menotti, A. Smerzi, and A. Trombettoni, New J. Phys. 5, 112 (2003); M. Kramer, C. Menotti, L. Pitaevskii and S. 
Stringari, Eur. Phys. J. D 27, 247 (2003); E. Taylor and E. Zaremba, Phys. Rev. A 68, 053611 (2003); N. P. Proukakis, N. G. Parker, C. F. Barenghi, and C. S. Adams, Phys. Rev. Lett. 93, 130408 (2004); D. Boers, C. Weiss, and M. Holthaus, Europhys. Lett. 67, 887 (2004); C. Kollath, U. Schollwock, J. von Delft, and W. Zwerger, Phys. Rev. A 71, 053606 (2005); Z. X. Liang, X. Dong, Z. D. Zhang, and B. A. Wu, Phys. Rev. A 78, 023622 (2008); A.-X. Zhang and J.-K. Xue, ibid. 80, $043617(2009)$.

[7] J. P. Martikainen and H. T. C. Stoof, Phys. Rev. A 69, 023608 (2004); C. C. Huang and W. C. Wu, ibid. A 72, 065601 (2005); S. D. Huber, E. Altman, H. P. Buchler, and G. Blatter, Phys. Rev. B 75, 085106 (2007).

[8] P. T. Ernst, S. Gotze, J. S. Krauser, K. Pyka, D. S. Luhmann, D. Pfannkuche, and K. Sengstock, Nature Phys. 6, 56 (2010).

[9] N. Marzari, A. A. Mostofi, J. R. Yates, I. Souza, and D. Vanderbilt, Rev. Mod. Phys. 84, 1419 (2012).

[10] A. Trombettoni and A. Smerzi, Phys. Rev. Lett. 86, 2353 (2001); A. Smerzi, A. Trombettoni, P. G. Kevrekidis, and A. R. Bishop, ibid. 89, 170402 (2002); G. L. Alfimov, P. G. Kevrekidis, V. V. Konotop, and M. Salerno, Phys. Rev. E 66, 046608 (2002).

[11] M. A. Porter, R. Carretero-González, P. G. Kevrekidis, and B. A. Malomed, Chaos 15, 015115 (2005); R. CarreteroGonzález, D. J. Frantzeskakis, and P. G. Kevrekidis, Nonlinearity 21, R139 (2008).

[12] A Maluckov, L. Hadžievski, B. A. Malomed, and L. Salasnich, Phys. Rev. A 78, 013616 (2008).

[13] G. Gligorić, A. Maluckov, Lj. Hažievski, and B. A. Malomed, Phys. Rev. A 78, 063615 (2008); J. Phys. B: At. Mol. Opt. Phys. 42, 145302 (2009).

[14] G. Gligorić, A. Maluckov, Lj. Hažievski, and B. A. Malomed, Phys. Rev. A 79, 053609 (2009).

[15] G. Gligorić, A. Maluckov, M. Stepić, L. Hadžievski, and B. A. Malomed, Phys. Rev. A 81, 013633 (2010); J. Phys. B: At. Mol. Opt. Phys. 43, 055303 (2010).

[16] K. Góral, K. Rzazewski and T. Pfau, Phys. Rev. A, 61, 051601 (R) (2000); L. Santos, G. V. Shlyapnikov, P. Zoller and M. Lewenstein, Phys. Rev. Lett. 85, 1791 (2000); K. Góral, L. Santos, and M. Lewenstein, Phys. Rev. Lett. 88, 170406 (2002); S. Yi, L. You, and H. Pu, Phys. Rev. Lett. 93, 040403 (2004); Y. Kawaguchi, H. Saito, and M. Ueda, ibid. 97, 130404 (2006); C. Menotti, C. Trefzger, and M. Lewenstein, ibid. 98, 235301 (2007); T. Lahaye, T. Pfau, and L. Santos, ibid. 104, 170404 (2010).

[17] A. Griesmaier, J. Werner, S. Hensler, J. Stuhler and T. Pfau, Phys. Rev. Lett. 94, 160401 (2005); J. Stuhler, A. Griesmaier, T. Koch, M. Fattori, T. Pfau, S. Giovanazzi, P. Pedri and L. Santos, ibid. 95, 150406 (2005); T. Lahaye, T. Koch, B. Frohlich, M. Fattori, J. Metz, A. Griesmaier, S. Giovanazzi, and T. Pfau, Nature 448, 672 (2007); A. Griesmaier, J. Phys. B: At. Mol. Opt. Phys. 40, 91 (2007); M. Fattori , G. Roati, B. Deissler, C. D'Errico, M. Zaccanti, M. Jona-Lasinio, L. Santos, M. Inguscio, and G. Modugno, Phys. Rev. Lett. 101, 190405 (2008); B. Pasquiou, G. Bismut, E. Maréchal, P. Pedri, L. Vernac, O. Gorceix, and B. Laburthe-Tolra, ibid. 106, 015301 (2011).

[18] T. Koch, T. Lahaye, J. Metz, B. Fröhlich, A, Griesmaier, and T. Pfau, Nature Physics 4, 218 (2008).

[19] T. Lahaye, C. Menotti, L. Santos, M. Lewenstein, and T. Pfau, Rep. Progr. Phys. 72, 126401 (2009).

[20] M. Lu, S. H. Youn, and B. L. Lev, Phys. Rev. Lett. 104, 063001 (2010); M. Lu, N. Q. Burdick, S. H. Youn, and B. L. Lev, ibid. 107, $190401(2011)$

[21] J. J. McClelland and J. L. Hanssen, Phys. Rev. Lett. 96, 143005 (2006); K. Aikawa, A. Frisch, M. Mark, S. Baier, A. Rietzler, R. Grimm, and F. Ferlaino, ibid. 108, 210401 (2012).

[22] J. Deiglmayr, A. Grochola, M. Repp, K. Mörtlbauer, C. Glück, J. Lange, O. Dulieu, R. Wester, and M. Weidemüller, Phys. Rev. Lett. 101, 133004 (2008); S. Ospelkaus, K.-K. Ni, G. Quéméner, B. Neyenhuis, D. Wang, M. H. G. de Miranda, J. L. Bohn, J. Ye, and D. S. Jin, Phys. Rev. Lett. 104, 030402 (2010).

[23] S. Müller, J. Billy, E. A. L. Henn, H. Kadau, A. Griesmaier, M. Jona-Lasinio, L. Santos, and T. Pfau, Phys. Rev. A 84, 053601 (2011); R. M. Wilson and J. L. Bohn, ibid. 83, 023623 (2011); M. Klawunn and L. Santos, Phys. Rev. A 80, 013611 (2009).

[24] A. Maluckov, G. Gligorić, Lj. Hadžievski, B. A. Malomed, and T. Pfau, Phys. Rev. Lett. 108, 140402 (2012).

[25] M. Machholm, A. Nicolin, C. J. Pethick, and H. Smith, Phys. Rev. A 69, 043604 (2004).

[26] A. Bühler and H. P. Büchler, Phys. Rev. A 84, 023607 (2011).

[27] A. Macia, D. Hufnag, F. Mazzanti, J. Boronat, and R. E. Zillich, arXiv:1209.1453.

[28] L. Santos, G. V. Shlyapnikov, and M. Lewenstein, Phys. Rev. Lett. 90, 250403 (2003); S. Ronen, D. C. E. Bortolotti, and J. L. Bohn, ibid. 98, 030406 (2007).

[29] W. S. Bakr, J. I. Gillen, A. Peng, S. Fölling, and M. Greiner, Nature 462, 74 (2009); J. F. Sherson, C. Weitenberg, M. Endres, M. Cheneau, I. Bloch, and S. Kuhr, Nature 467, 68 (2010).

[30] I. Shammass, S. Rinott, A. Berkovitz, R. Schley, and J. Steinhauer, arXiv:1207.3440.

[31] J. Steinhauer, N. Katz, R. Ozeri, N. Davidson, C. Tozzo, and F. Dalfovo, Phys. Rev. Lett. 90, 060404 (2003).

[32] O. M. Braun and Y. S. Kivshar, Phys. Rep. 306, 1 (1998); D. Hennig and Tsironis, ibid. 307, 333 (1999).

[33] D. O’Dell, S. Giovanazzi, G. Kurizki, and V. M. Akulin, Phys. Rev. Lett. 84, 5687 (2000); S. Giovanazzi, D. O’Dell, and G. Kurizki, Phys. Rev. A 63, 031603 (2001); I. Papadopoulos, P. Wagner, G. Wunner, and J. Main, ibid. 76, 053604 (2007).

[34] S. A. Akhmanov, D. P. Krindach, A. V. Migulin, A. P. Sukhorukov, and R. V. Khokhlov, IEEE J. Quantum Electron. 4, 568 (1968).

[35] W. Królikowski, O. Bang, J. J. Rasmussen, and J. Wyller, Phys. Rev. E 64, 016612 (2001); O. Bang, W. Królikowski, J. Wyller, and J. J. Rasmussen, ibid. 66, 046619 (2002); W. Królikowski, O. Bang, J. J. Rasmussen, and J. Wyller, Opt. Express 13, 435 (2005).

[36] C. Rotschild, B. Alfassi, O. Cohen, and M. Segev, Nature Phys. 2, 769 (2006); A. Dreischuh, D. N. Neshev, D. E. Petersen, O. Bang, and W. Królikowski, Phys. Rev. Lett. 96, 043901 (2006). 
[37] C. Conti, M. Peccianti, and G. Assanto, Phys. Rev. Lett. 91, 073901 (2003).

[38] E. A. Ulnatir, G. I. Stegeman, D. Michaelis, C. H. Lange, and F. Lederer, Phys. Rev. Lett. 90, 253903 (2003).

[39] T. H. Stix, Theory of plasma waves (Springer-Verlag New York, Inc. 1992). 